\title{
Surgical Procedure of Balloon Kyphoplasty and Cement Injection in Osteoporotic Vertebral Body Fractures
}

\section{Mohsen Ghiassi*}

Department of Spine Surgery, Helios Clinic, Lengerich, Germany

\section{Clinical Image}

Osteoporosis is a common, often undiagnosed disease in elderly and multimorbid patients. A reduction in bone mass and alteration of the bone structure leads, above all, to vertebral body fractures resulting in immobilization and long-term care. Early prevention as well as timely diagnosis and effective therapy are therefore of

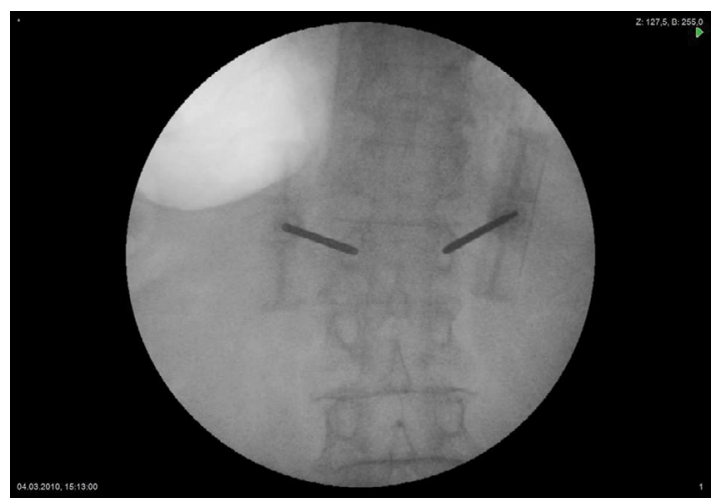

Figure 1: Placing working sleeves.

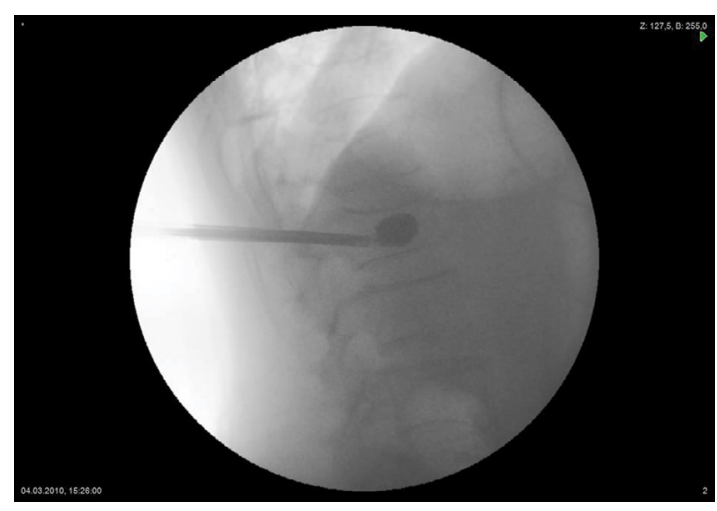

Figure 2: Filling the balloon with contrast agent.

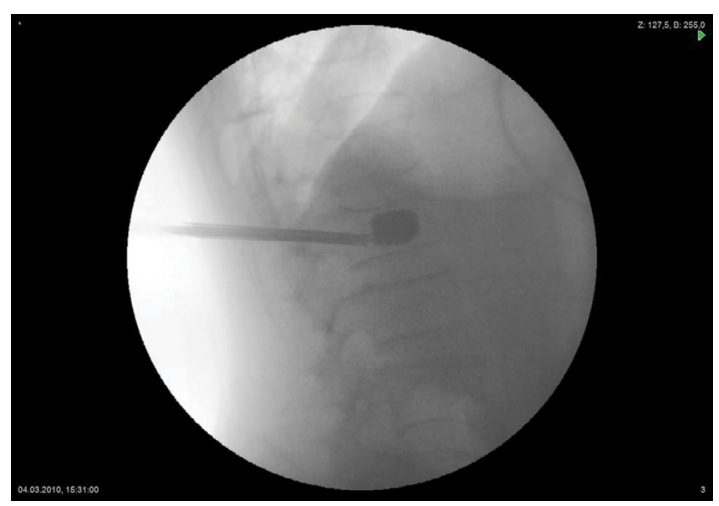

Figure 3: Reaching the good height of the vertebral body. great importance. Operative stabilization using a minimally invasive procedure called balloon kyphoplasty has been one of the established methods for years. Balloon kyphoplasty provides a good and efficient pain relief with faster mobilization of patients and thus avoiding a need for care mit fast return the patients home. The surgery lasts between 30 to 60 minutes under general anesthesia. In some cases it is also possible in local anesthesia (Figures 1-8).

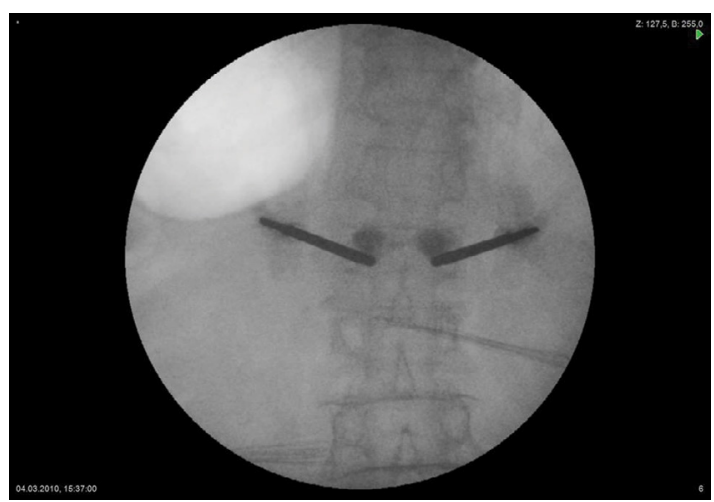

Figure 4: Injection the cement in the vertebral body.

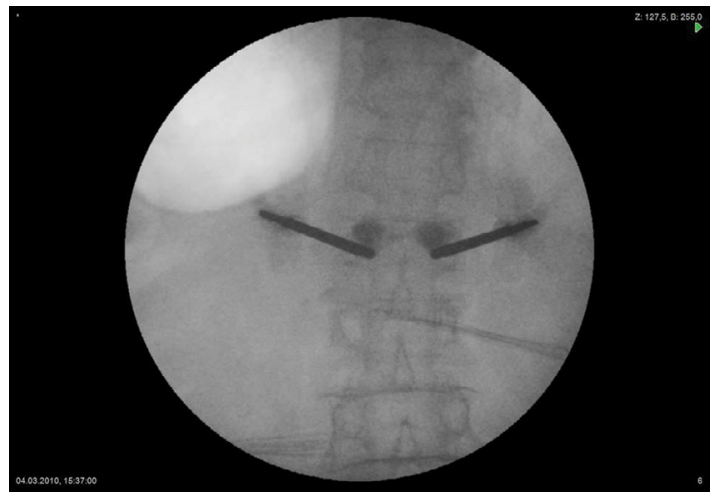

Figure 5: Spraying the cement bilateral.

*Corresponding author: Mohsen Ghiassi, Medical Director, Department of Spine Surgery, Helios Clinic, Lengerich, Germany, Tel: + 4917650157865; E-mail: ghiassi@arcor.de

Received August 23, 2018; Accepted September 03, 2018; Published September 06, 2018

Citation: Ghiassi M (2018) Surgical Procedure of Balloon Kyphoplasty and Cement Injection in Osteoporotic Vertebral Body Fractures. J Spine S7: 014. doi: 10.0142/2165-7939.S7-014

Copyright: ( 2018 Ghiassi M. This is an open-access article distributed under the terms of the Creative Commons Attribution License, which permits unrestricted use, distribution, and reproduction in any medium, provided the original author and source are credited. 
Citation: Ghiassi M (2018) Surgical Procedure of Balloon Kyphoplasty and Cement Injection in Osteoporotic Vertebral Body Fractures. J Spine 7: 014. doi: $10.0142 / 2165-7939.1000014$

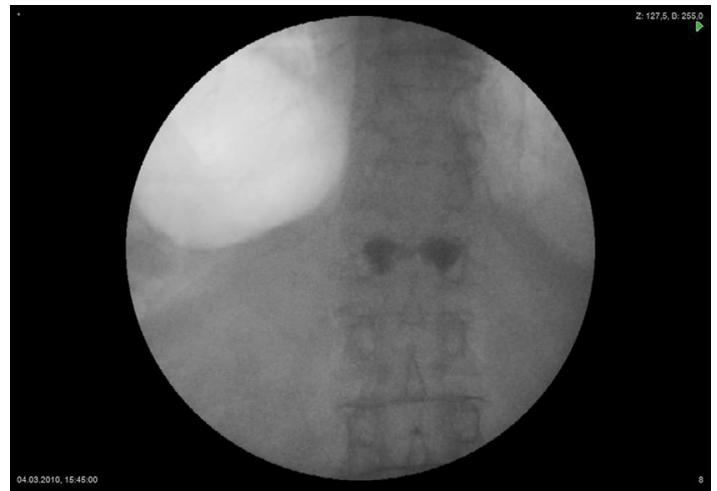

Figure 6: Cement layer after removal of working sleeves.

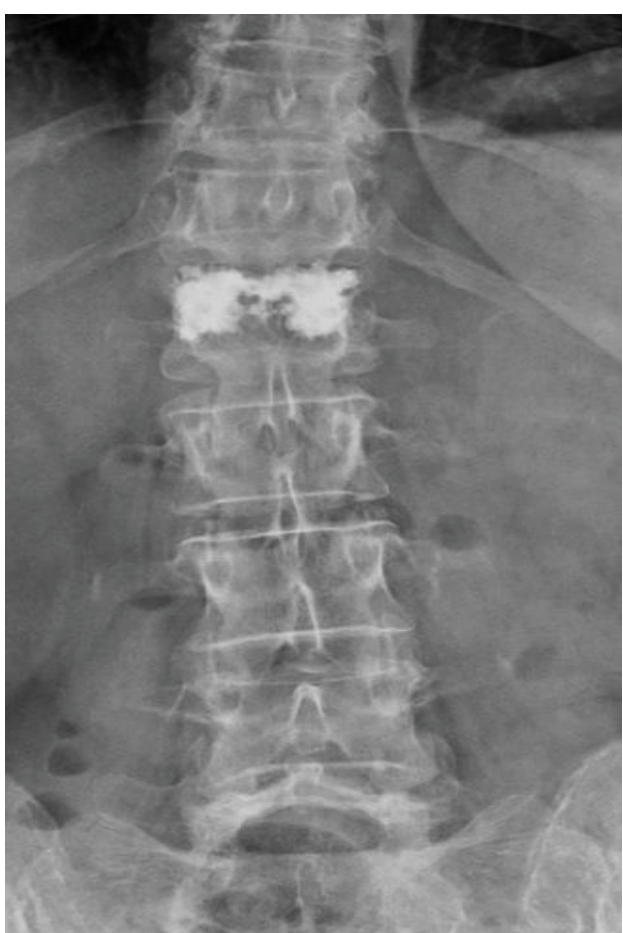

Figure 7: X-ray image in A.P. after ballon kyphoplasty.

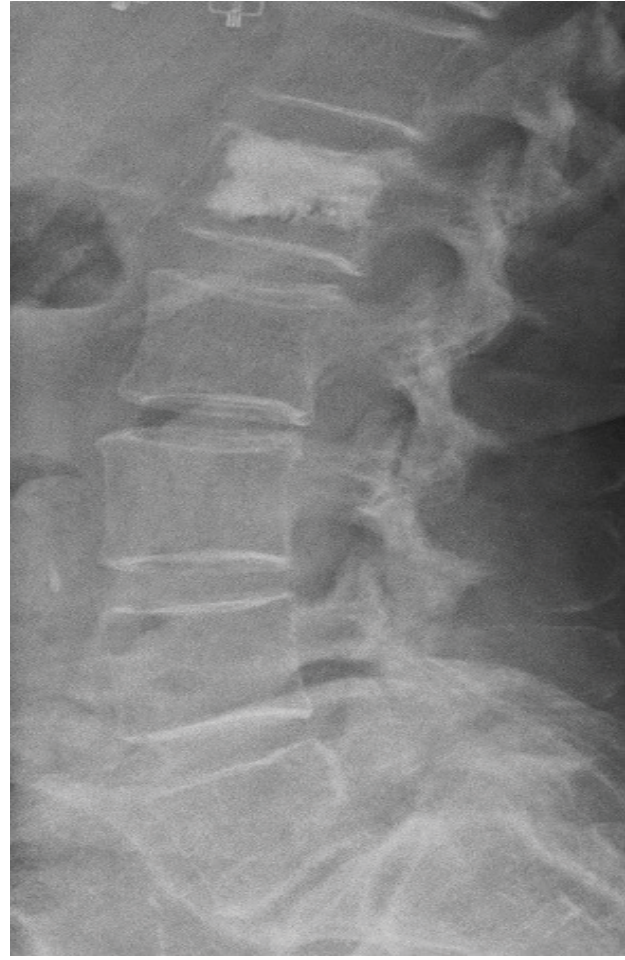

Figure 8: X-ray image laterally after the operation.

This article was originally published in a special issue, Minimally Invasive Spine Surgery -II handled by Editor(s). Anthony T. Yeung, M.D. Clinical professor, University of New Mexico School of Medicine Associate Desert Institute for Spine Care, Phoenix, Arizona, Executive director International Intradiscal therapy Society Phoenix, Arizona 